\title{
rSPECT: A Compact Gamma Camera Based SPECT System for Small-Animal Imaging
}

\author{
Eduardo Lage ${ }^{1}$, José L. Villena ${ }^{1}$, Gustavo Tapias ${ }^{1}$, I. Vidal-Migallón, Mónica Abella ${ }^{1}$, Alejandro Sisniega ${ }^{1}$, Naira P. \\ Martínez ${ }^{3}$, Doménec Ros ${ }^{3,4}$, Manuel Desco ${ }^{1,2}$, Juan J. Vaquero ${ }^{1}$, Senior Member IEEE
}

\begin{abstract}
We have developed a compact and modular gamma camera system and assessed its performance when used on a small-animal SPECT prototype (rSPECT). Each camera consists of a Hamamatsu H-8500 position sensitive photomultiplier tube coupled to a $30 \times 30 \mathrm{NaI}(\mathrm{TI})$ scintillator array $(1.4 \mathrm{~mm} \times 1.4 \mathrm{~mm} \times$ $6 \mathrm{~mm}$ crystal size) and electronics for pre-processing and matching the detector signals to an in-house developed data acquisition system. The camera components are enclosed in a lead-shielded case with a receptacle to insert the collimators (parallel-hole or pinhole with different tungsten apertures). System performance has been assessed for a low energy high resolution parallel-hole collimator (LEHR), and for a $0.75 \mathrm{~mm}$ pinhole collimator with $60^{\circ}$ aperture angle. In this paper we present details on the system implementation and results of performance measurements, as well as first tomographic images on phantoms and animals. This SPECT was conceived for compactness and cost-effective routine small-animal imaging, and acquisitions of living mice and rats carried out with the system demonstrate its ability to provide useful high-resolution images for in vivo research.
\end{abstract}

Index Terms Single Photon Emission Computed Tomography (SPECT), small-animal imaging, gamma camera.

\section{INTRODUCTION}

$\mathrm{T}$ HE development of high-performance SPECT imagers dedicated to small animals has been possible due to the use of pinhole collimation [1-4]. This configuration results in good sensitivity with a spatial resolution greater than the detector intrinsic resolution due to the magnification effect when the object is placed close to the pinhole. The tradeoff is, however, a reduced field of view proportional to the source-to-pinhole distance. Several approaches and strategies have been adopted in commercial systems in order to achieve greater sensitivities

Manuscript received November 13, 2009.

This work is partially funded by the CD TEAM project, CENIT Program, Spanish Ministerio de Industria and with grants from the Ministerio de Educación y Ciencia, Projects TEC2007 64731/TCM, TEC2008 06715 C02 01, SAF2009 08076 and the RECAVA RETIC Network.

E. Lage, J. J. Vaquero, A. Sisniega, G. Tapias, J. L. Villena, I.Vidal Migallón and $\mathrm{M}$ Desco are with the Unidad de Medicina y Cirugía Experimental, Hospital General Universitario Gregorio Marañon, Madrid, Spain. (Eduardo Lage email: elage@hggm.es, Juan José Vaquero e mail: juanjo@mce.hggm.es).

D. Ros and N. P. Martínez are with the Unidad de Biofísica y Bioingeniería, Universidad de Barcelona IDIBAPS. Spain.

M. Desco is also with the CIBERSAM, Madrid, Spain.

D. Ros is also with CIBER BBN, Zaragoza, Spain and good spatial resolution $(<0.5$ to $2 \mathrm{~mm}$ or less $)$ while maintaining a Field of View (FOV) appropriate for the application. Most of them make use of multi-pinhole collimation with high magnification values, combined with big-area detectors with moderate intrinsic resolution, such as human gamma-cameras [5-7]. Although these systems provide a wide range of possibilities for translational research, most common protocols encountered in practice can also be accomplished using more cost-effective solutions. Among them, tomographic systems dedicated to small-animals [8-12] and planar systems using similar technology, either dedicated to small-animals [13-15] or clinical purposes [16-18] have been extensively investigated.

In this work we present a compact and modular gamma camera system based on small-area and high resolution detectors which can be equipped with interchangeable collimators (parallel-hole or pinhole with different apertures). We assessed the performance of this detector by assembling a small-animal SPECT prototype (rSPECT) using two of these cameras. The detector performance has been evaluated in terms of spatial resolution, uniformity, sensitivity and energy resolution. Micro hot-rod phantom studies were also carried out and in vivo animal studies were conducted to investigate the system performance in real small-animal SPECT applications. The main purpose of this work was to evaluate the feasibility of these cameras to be used as the basic detector module for a low-cost SPECT/CT system for small-animal imaging.

\section{MATERIALS AND METHODS}

\section{A. Gamma Cameras}

Each gamma-camera consist of a Hamamatsu H-8500 position sensitive photomultiplier tube (PS-PMT) with $\approx 45$ $\mathrm{mm} \times 45 \mathrm{~mm}$ active area, a $30 \times 30 \mathrm{NaI}$ (Tl) scintillator array with elements of $1.4 \mathrm{~mm} \times 1.4 \mathrm{~mm} \times 6 \mathrm{~mm}(1.6 \mathrm{~mm}$ pitch $)$ and the electronics needed for matching the detector signals to an in-house developed data acquisition system. The aforementioned electronics consists of a compact PCB stack directly attached to the PS-PMT sockets. We included a symmetric charge division circuit [19] wich combines the 64 output signals of the PMT to obtain 16 signals, amplification and shaping stages for them, an additional stage for the last dynode signal of the PMT and the HV supply. 


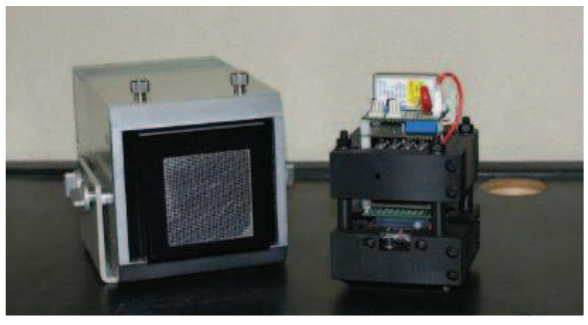

(a)

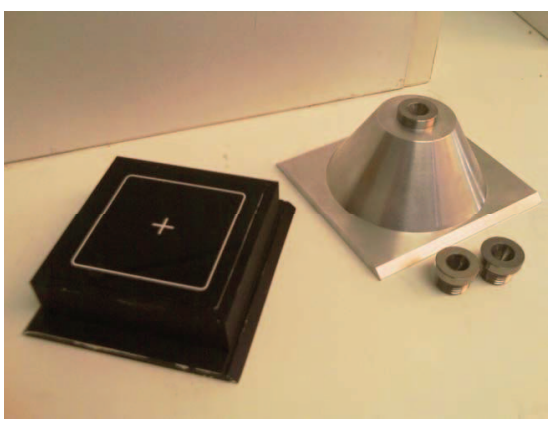

(b)

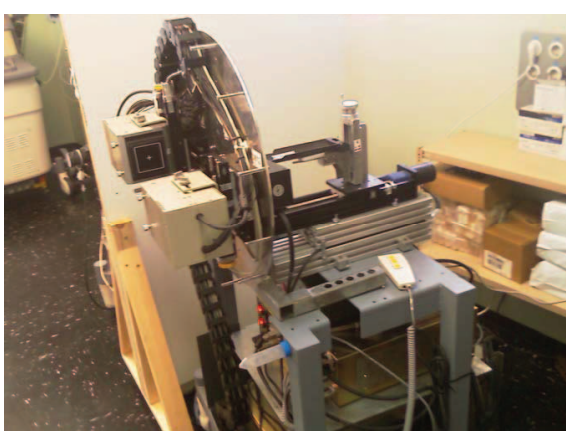

(c)

Figure 1. (a) Lead shielded case equipped with a parallel hole collimator (left) and SPECT detector (right), (b) LEHR parallel hole collimator (left) and pinhole collimator with different apertures (right) .(c) r SPECT prototype

The scintillator array, the PMT and the electronics are assembled in a black-delrin enclosure (see Figure 1.a) which fit in a lead-covered case. This enclosure enables the attachment of the cameras to a gantry with a mechanical solution for easily changing the collimator depending on the study requirements. The camera configurations evaluated on this work (Figure 1.b) are based on a) low-energy and high-resolution parallel-hole collimator (LEHR) with 1.2 $\mathrm{mm}$ hexagonal holes, $20 \mathrm{~mm}$ thick, and with a $0.2 \mathrm{~mm}$ septal thickness, and b) pinhole collimator with $42 \mathrm{~mm}$ detector-to-pinhole distance, $10 \mathrm{~mm}$ thick lead-shielding, $60^{\circ}$ aperture angle and interchangeable tungsten inserts that screw in and out of the collimator (a $0.75 \mathrm{~mm}$ aperture was used on this work).

\section{B. Acquisition Electronics}

Data acquisition system of the rSPECT (Figure 2) consists of a master module implemented using a CPLD which is in charge of the generation and distribution of trigger signals, 2 x 16-channel ADC cards (12-bit resolution) and a 32-bit digital I/O PCI card (NI-6533-DIO$32 \mathrm{HS}$, National Instruments) for handling data transmission into a computer.

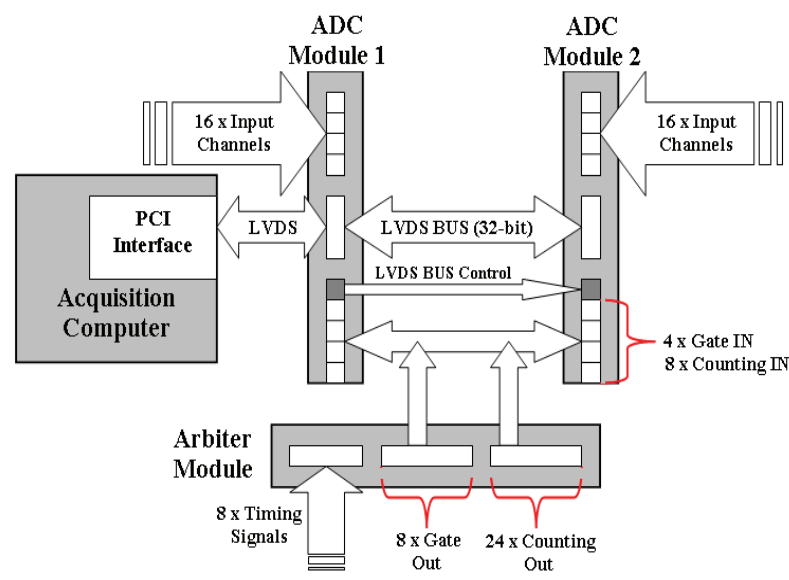

Figure 2. rSPECT data acquisition system components and topology. The system contains an arbiter module, two ADC modules and a digital I/O PCI interface. ADC modules and PCI card are connected using a 32 bit LVDS bus.
Additionally, we included 8 general purpose 12-bit counters per ADC card which are transmitted with each digitized event. ADC cards and the digital I/O PCI interface are connected using a high-speed 32-bit LVDS BUS. Due to this architecture, ADC modules are also daisy-chained in order to access the LVDS BUS coherently. Each ADC channel and data counter includes a 4 bit header used as an identifier in the analysis of the data frames.

Last dynode signals of the detectors are fed into pulse discriminator circuits located in the arbiter module. These circuits generate timing signals which allow detecting and discriminating photons as a function of its energy. After the detection of a valid event, trigger signals are generated and transmitted to the ADC modules to start the digitization of the position signals necessary to characterize the event. These position signals are integrated for a 300 ns period, digitized and sent to the $\mathrm{PC}$ in order of arrival. The current value of the counters included in each ADC card is sent together with the data frame for each event acquired, providing synchronization information such as the detectors position, global time or gating signals.

\section{C. rSPECT Prototype}

The small-animal SPECT prototype is based on two opposite gamma cameras mounted on a motorized gantry (Figure 1.c). The mechanical system of this gantry consists of 6 motorized movement axes controlled by a computer by means of RS-232 communication and a set of digital servo drives (ISCM-4805, Technosoft) interconnected using a CAN bus. Four motorized axes are mounted on the rotatory stage of the gantry allowing detectors to be displaced on the tangential and radial directions for the adjustment of radius and center of rotation. A sample-holder enables the movement of the sample along the axial direction.

The entire system is controlled by a single PC running Linux operating system. Control software written in ANSI $\mathrm{C}$ is in charge of synchronizing data acquisition with system movements in order to implement the different acquisition protocols. During scans, this software receives the acquired data together with synchronism information from the PCI interface and processes them to correct pedestal values of ADC channels, calculate the position of interaction and energy deposited by the photons and generate list-mode files containing this information and 
synchronization events constructed from the values included in the counters.

Scan planning, data processing, image reconstruction and displaying are managed from a remote computer which runs an IDL-based (ITT Visual Information Solutions) console that also provides user interface and analysis tools [20].

\section{Calibration and Data Processing}

After acquisition, list-mode files are processed by the user interface and converted into projections. This process includes the correction of the camera non-uniformity, nonlinearity, energy factors and other effects, such as radioactive decay or detectors alignment. The main correction factors are obtained from a field flood acquisition. The flood source used for the evaluation consisted of a plastic container ( $8 \mathrm{~mm}$ thick) sufficiently large to cover the entire detectors filled with $2 \mathrm{mCi}$ of ${ }^{99} \mathrm{~m}$ Tc. After intrinsic flood image acquisition $(>10$ kcounts per pixel), a lookup table is created in order to associate each scintillator pixel with a region on the PS-PMT, thus intrinsically correcting spatial linearity of the cameras. Based on that table, the pulse-height spectrum is generated and energy-correction factors are obtained to align and scale the spectrum of each pixel to a common reference. This information is used to set the energy window for image acquisition. After correcting the acquired flood source using the energy-correction factors, we compared the number of counts recorded within each pixel with the mean pixel value of the flood source. With these data, a non-uniformity correction file with the relative efficiency of each image element is obtained. Each subsequently acquired image is corrected using the energy and uniformity correction values which were saved in a single file.

\section{E. Pinhole and Parallel hole SPECT Reconstruction}

Analytical and statistical approaches were employed for image reconstruction. With regards to analytical methods, we employed a standard filtered backprojection algorithm for parallel-hole based acquisitions, and the FDK algorithm [21] for pinhole based acquisitions. The statistical techniques used were based on an iterative ordered subsetsexpectation maximization (OSEM) algorithm [22]. The algorithms employed for the parallel collimator were adapted to the small animal SPECT scanner from 2D [23] and 3D [24] algorithms previously developed for clinical cameras. The algorithm for the pin-hole collimator was also an in-house version of [24] adapted to this specific geometry. All reconstruction iterative algorithms included correction of the spatial variant collimator response.

\section{F. Performance Evaluation}

The flood field uniformity was measured with the aforementioned flood source. The energy resolution was obtained for each usable pixel from the field flood images obtained during the calibration process. A $5 \times 5 \times 8 \mathrm{~mm}^{3}$ square phantom filled with aqueous ${ }^{99 \mathrm{~m}} \mathrm{Tc}$ was used to evaluate the cameras sensitivity at different source-to collimator distances following the guidelines described on NEMA specifications [25].
Planar measurements were assessed for both cameras and collimators to determine the full-width at half-maximum (FWHM) of the line spread functions (LSFs). A single 0.3$\mathrm{mm}$ inner diameter glass capillary source filled with aqueous ${ }^{99 \mathrm{~m}} \mathrm{Tc}$ and axially aligned with the detectors was used to evaluate the spatial resolution of the cameras at different source-to-collimator distances. For these experiments the line source and the cameras were first leveled axially in such a way that the image was projected in the central pixels of both detectors. On the first measurement the source-to-collimator distance was $10 \mathrm{~mm}$ with both collimator-types. Cameras were stepped in 5-mm increments using the motorized stages included on the gantry until reach a source-to-collimator distance of $45 \mathrm{~mm}$. Projections were acquired for 120 seconds at each camera position using a $20 \%$ symmetric energy window. FWHM spatial resolution of the planar images was determined by fitting a Gaussian function to the count profiles and by direct calculation from the profile data using a linear interpolation between the points above and below the halfmaximum value.

\section{G. SPECT Imaging}

The spatial resolution has been evaluated in SPECT mode using a micro-Derenzo phantom (Ultra-Micro Hot Spot Phantom, Data Spectrum Corporation, Hillsborough, NC). This phantom has six sectors with rod diameters of 2.4, 2.0, $1.7,1.35,1.0$, and $0.75 \mathrm{~mm}$. Center-to center spacing of channels is two times the rod diameter. The phantom was filled with $4.5 \mathrm{mCi}$ of ${ }^{99 \mathrm{~m}} \mathrm{Tc}$ and acquired for two hours using the pinhole collimators equipped with the $0.75 \mathrm{~mm}$ apertures. A total of 120 projections were acquired over a $360^{\circ}$ span with a $33.5-\mathrm{mm}$ radius of rotation (ROR).

In order to assess the possibilities of the system for in vivo imaging the rSPECT has been tested in several common imaging protocols based on ${ }^{99 \mathrm{~m}}$ Tc-MDP tracer. Whole-body bone scans of a $154 \mathrm{~g}$ rat and of a $24 \mathrm{~g}$ mouse were acquired with parallel-hole and pinhole collimators respectively. The rat was injected with $6.3 \mathrm{mCi}$ and the mouse with $5.2 \mathrm{mCi}$ of the aforementioned radiopharmaceutical. After a $2 \mathrm{~h}$ uptake, 60 projections were acquired over a $360^{\circ}$ span (ROR $37.2 \mathrm{~mm}$ and 32.1 $\mathrm{mm}$ for rat and mouse respectively) on each of the four axial positions that composed the scans (total scan time was $2 \mathrm{~h}$ in both cases).

\section{RESULTS}

\section{A. Performance Evaluation}

The performance of the implemented gamma cameras has been characterized in terms of spatial resolution, sensitivity, uniformity and energy resolution.

Values of energy resolution at various locations and integral uniformity over the central (CFOV) and useful (UFOV) fields of view are presented in Table I.

The results of the planar resolution measurements as a function of source-to-collimator distance are shown in Figure 3. Figure 4 shows the camera sensitivity with parallel-hole and pinhole collimators $(0.75 \mathrm{~mm}$ aperture $)$ 
attached. Differences in spatial resolution and sensitivity between both detectors were negligible.

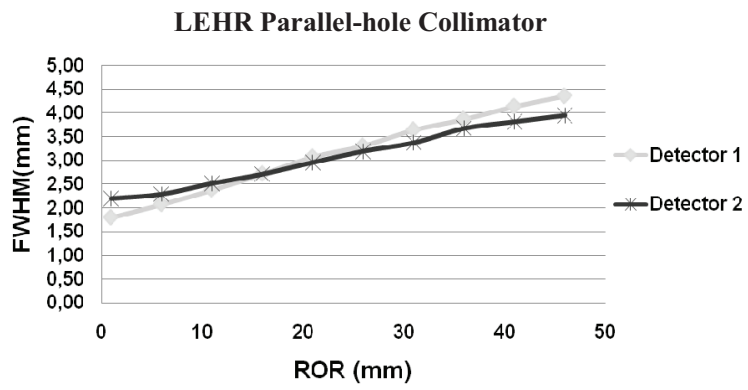

Pinhole Collimator (0.75 mm Aperture)

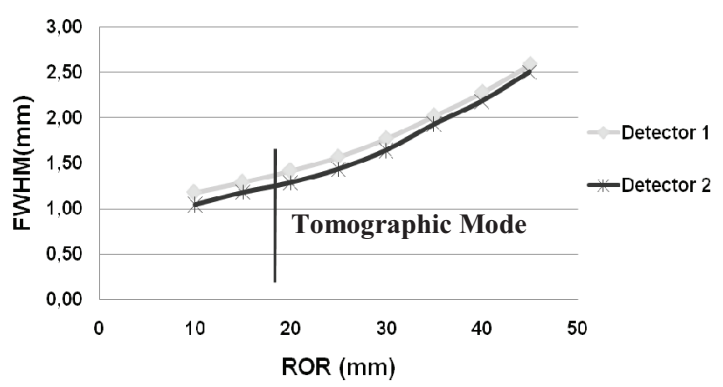

Figure 3. Planar spatial resolution (FWHM) as a function of the selected radii of rotation (ROR). Measurements were done using the parallel hole collimators (up) and the pinhole collimators (bottom) equipped with the $0.75 \mathrm{~mm}$ apertures. In both cases a $20 \%$ symmetric energy window was used.

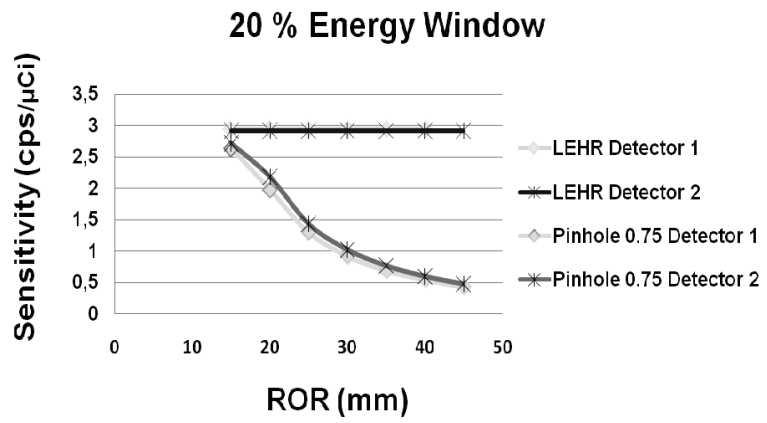

Figure 4. Sensitivity of the cameras as a function of the selected radii of rotation (ROR). Measurements were done using the parallel hole collimators and the pinhole collimators equipped with the $0.75 \mathrm{~mm}$ apertures.

\section{B. SPECT Phantom Imaging}

A transverse slice through the reconstructed volume is shown in Figure 5, where the largest four sectors of the phantom are easily resolved. Image shown in Figure 5 was reconstructed using 3-D OSEM with 8 subsets and 16 iterations (voxel size $0.4 \times 0.4 \times 0.8 \mathrm{~mm}^{3}$ ).

\section{In vivo Imaging}

Figure 6 ( $\mathrm{a}$ and $\mathrm{b}$ ) shows bone studies obtained with ${ }^{99 \mathrm{~m}} \mathrm{Tc}-$ MDP. The mouse image was obtained with the pinhole collimator and reconstructed using the FDK algorithm (pixel size $1.1 \mathrm{~mm}$, slice thickness $1.1 \mathrm{~mm}$ ) and processed with a Butterworth filter (cutoff 0.35/pixel, order 12).

TABLE I

INTEGRAL UNIFORMITY (\%) AND ENERGY RESOLUTION (\%) AT $140 \mathrm{KEV}$

Camera 1 Camera 2

\begin{tabular}{ccc}
\hline $\begin{array}{c}\text { Intrinsic } \\
\text { Non-Uniformity } \\
\text { (UFOV) }\end{array}$ & $11.3 \%$ & $9.8 \%$ \\
\hline $\begin{array}{c}\text { Intrinsic } \\
\text { Non-Uniformity } \\
\text { (CFOV) }\end{array}$ & $7.9 \%$ & $7.7 \%$ \\
\hline $\begin{array}{c}\text { Energy Resolution } \\
\text { (Average, UFOV) }\end{array}$ & $937 \%$ & $10.4 \%$ \\
\hline $\begin{array}{c}\text { Energy Resolution } \\
\text { (Best Crystal, UFOV) }\end{array}$ & $7.8 \%$ & $8.1 \%$ \\
\hline $\begin{array}{c}\text { Energy Resolution } \\
\text { (Worst Crystal, } \\
\text { UFOV) }\end{array}$ & $17.4 \%$ & $16.2 \%$ \\
\hline
\end{tabular}

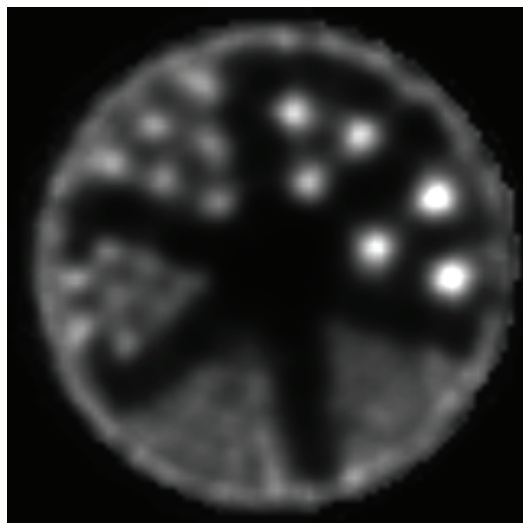

Figure 5. Micro Derenzo phantom axial slice. Hot rods of 1.35 $\mathrm{mm}$ can be resolved in the image. $4.5 \mathrm{mCi}$ of ${ }^{99 \mathrm{~m}} \mathrm{Tc}$ was added to the phantom. The image was acquired in 120 min using the pinhole collimator.

The rat study was acquired with parallel-hole collimators and reconstructed using 2-D OSEM with 8 iterations and 64 subsets (pixel size $0.4 \mathrm{~mm}$, slice thickness $0.8 \mathrm{~mm}$ ).

\section{SUMMARY AND CONCLUSIONS}

A modular and versatile gamma camera technology has been developed based on small-field-of-view, high-spatialresolution and modular detectors. Detector performance has been evaluated in terms of spatial and energy 


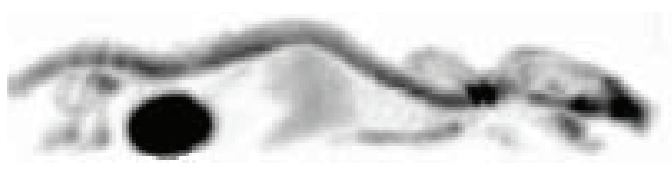

(a)

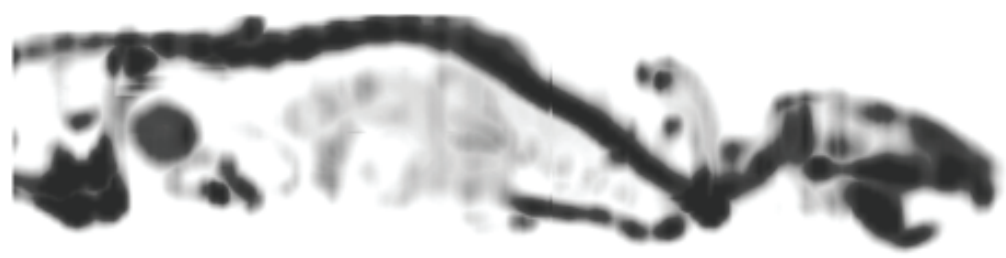

(b)

Figure 6. (a) MIP render of a $24 \mathrm{~g}$ mouse bone scan $\left({ }^{99 \mathrm{~m}} \mathrm{Tc}\right.$ MDP). Image was acquired using pinhole collimators, data were reconstructed using FDK algorithm (b) MIP render of a $154 \mathrm{~g}$ rat bone scan $\left({ }^{99 \mathrm{~m}} \mathrm{Tc}\right.$ MDP). Image was acquired using parallel hole collimators, data were reconstructed using 2 D OSEM.

resolution, sensitivity and uniformity, showing good characteristics for the use of this technology in a planar system such as a hand-held camera, similar to that reported on [16]. The possibilities of these detectors as a component of a small-animal SPECT system have been evaluated by implementing a dedicated tomograph.

The goal of the tests carried out was to evaluate the possibility of using high resolution detectors combined with low magnification factors in a compact SPECT/CT system for small-animals with a configuration similar to that reported in [26]. Although the rSPECT prototype has been constructed for compactness and cost-effective routine small-animal imaging, studies of living mice and rats obtained with this system demonstrate its ability to provide useful high resolution images for in vivo research.

\section{ACKNOWLEDGMENT}

The authors thanks SEDECAL Engineering department for their support during the system development. This work is supported by the Spanish Ministerio de Ciencia e Innovación (TEC-2008-06715-C02-01, TEC-200764731/TCM and SAF 2009-08076), Ministerio de Industria (CDTEAM, Programa CENIT) and the RECAVA-RETIC Network.

\section{REFERENCES}

[1] R. J. Jaszczak, L. JianYing, W. Huili, M. R. Zalutsky, and R. E. Coleman, "Pinhole colimation for ultrahigh resolution small field of view SPECT," Physics in Medicine and Biology, vol. 39, pp. 425 437, 1994

[2] K. Ogawa, T. Kawade, K. Nakamura, A. Kubo, and T. Ichihara, "Ultrahigh resolution pinhole SPECT for small animal study," IEEE Transactions on Nuclear Science, vol. 45, pp. 3122 3126, 1998.

[3] D. Paix, "Pinhole Imaging of Gamma Rays," Physics in Medicine and Biology, vol. 12, pp. 489 500, 1967.

[4] L. R. Furenlid, D. W. Wilson, Y. Chen, H. Kim, P. J. Pietraski, M. J. Crawford, and H. H. Barrett, "FastSPECT II: A Second Generation High Resolution Dynamic SPECT Imager," IEEE Transactions on Nuclear Science, vol. 51, pp. 631 635, 2004.

[5] J. B. A. Habraken, K. de Bruin, M. Shehata, J. Booij, R. B. Berthe, L. F. van Eck Smit, and E. B. Sokole, "Evaluation of High Resolution Pinhole SPECT Using a Small Rotating Animal," The Journal of Nuclear Medicine, vol. 42, pp. 1863 1869, 2001.

[6] F. Beekman and B. Vastenhouw, "Submillimeter Total Body Murine Imaging with U SPECT I," The Journal of Nuclear Medicine, vol. 48, pp. 487 493, 2007.

[7] F. van der Have, B. Vastenhouw, R. M. Ramakers, W. Branderhorst, J. O. Krah, C. Ji, S. G. Staelens, and F. J.
Beekman, "U SPECT II: An Ultra High Resolution Device for Molecular Small Animal Imaging." vol. 50, 2009, pp. 599605.

[8] L. R. MacDonald, B. E. Patt, J. S. Iwanczyk, B. M. W. Tsui, Y. Wang, E. C. Frey, D. E. Wessell, P. D. Acton, and H. F. Kung, "Pinhole SPECT of Mice Using the LumaGEM Gamma Camera," IEEE Transactions on Nuclear Science, vol. 48, pp. $830836,2001$.

[9] D. P. McElroy, L. R. MacDonald, F. J. Beekman, y. Wang, B. E. Patt, J. S. Iwanczyk, B. M. W. Tsui, and E. J. Hoffman, "Performance Evaluation of A SPECT: A High Resolution Desktop Pinhole SPECT System for Imaging Small Animals," IEEE Transactions on Nuclear Science, vol. 49, pp. 21392147 , 2002.

[10] T. Y. Song, Y. Choi, J. H. Jung, B. J. Min, K. J. Hong, Y. S. Choe, K. Lee, and B. Kim, "Performance Amelioration for Small Animal SPECT Using Optimized Pinhole Collimator and Image Correction Technique," IEEE Transactions on Nuclear Science, vol. 52, pp. 1396 1400, 2005.

[11] A. Del Guerra, A. Bartoli, N. Belcari, D. Herbert, A. Motta, A. Vaiano, G. Di Domenico, N. Sabba, E. Moretti, G. Zavattini, M. Lazzarotti, L. Sensi, M. Larobina, and L. Uccelli, "Performance evaluation of the Fully Engineered YAP (S)PET Scanner for Small Animal Imaging," IEEE Transactions on Nuclear Science, vol. 53, pp. 1078 1083, 2006.

[12] A. G. Weisenberger, R. Wojcik, E. L. Bradley, P. Brewer, S. Majewski, j. Qian, A. Ranck, M. S. Saha, K. Smith, M. F. Smith, and R. E. Welsh, "SPECT CT System for Small Animal Imaging," IEEE Transactions on Nuclear Science, vol. 50, pp. 7479,2003

[13] G. Loudos, S. Majewski, R. Wojcik, A. G. Weisenberger, N. Sakellios, K. Nikita, N. Uzunoglu, P. Bouziotis, S. Xanthopoulos, and A. Varvarigou, "Performance Evaluation of a Dedicated Camera Suitable for Dynamic Radiopharmaceuticals Evaluation in Small Animals," IEEE Transactions on Nuclear Science, vol. 54, pp. 454 460, 2007.

[14] E. L. Bradley, J. Cella, S. Majewski, V. Popov, J. Qian, M. S. Saha, M. F. Smith, A. G. Weisenberger, and R. E. Welsh, "A Compact Gamma Camera for Biological Imaging," IEEE Transactions on Nuclear Science, vol. 53, 2003.

[15] S. R. Meikle, P. Kench, A. G. Weisenberger, R. Wojcik, M. F. Smith, S. Majewski, S. Eberl, R. R. Fulton, A. B. Rosenfeld, and M. J. Fulham, "A Prototype Coded Aperture Detector for Small Animal SPECT," IEEE Transactions on Nuclear Science, vol. 49, 2002.

[16] P. D. Olcott, F. Habte, A. M. Foudray, and C. S. Levin, "Performance Characterization of a Miniature, High Sensitivity Gamma Ray Camera," IEEE Transactions on Nuclear Science, vol. 54, pp. 1492 1497, 2007.

[17] M. J. Moré, P. J. Goodale, S. Majewski, and M. B. Williams, "Evaluation of Gamma Cameras for Use in Dedicated Breast Imaging," IEEE Transactions on Nuclear Science, vol. 53, pp. $26752679,2006$.

[18] M. P. Tornai, C. N. Brzymialkiewicz, M. L. Bradshaw, J. E. Bowsher, B. E. Patt, J. S. Iwanczyk, J. Li, and L. R. MacDonald, "Comparison of Compact Gamma Cameras With 1.3 and $2.0 \mathrm{~mm}$ Quantized Elements for Dedicated Emission Mammotomography," IEEE Transactions on Nuclear Science, vol. 52, pp. 1251 1256, 2005. 
[19] P. D. Olcott, J. A. Talcott, C. S. Levin, F. Habte, and A. M. K. Foudray, "Compact Readout Electronics for Position Sensitive Photomultiplier Tubes," IEEE Transactions on Nuclear Science, vol. 52, pp. 21 27, 2005.

[20] J. Pascau, J. J. Vaquero, M. Abella, R. Cacho, E. Lage, and M. Desco, "Multimodality workstation for small animal image visualization and analysis," Mol. Imaging Biol., vol. 8, pp. 97 98, 2006.

[21] L. A. Feldkamp, L. C. Davis, and J. W. Kress, "Practical cone beam algorithm," J Opt Soc Am, vol. 1, pp. 612 619, 1984.

[22] H.M. Hudson, and R.S Larkin, "Accelerated image reconstruction using ordered subsets of projection data", IEEE Trans Med Imaging. vol. 13, pp 601 609, 1994.
[23] D. Pareto, A. Cot, J. Pavia, C. Falcon, I. Juvells, F. Lomena and D. Ros, "Iterative reconstruction with compensation of the spatial variant fan beam collimator response in neurotransmission SPET imaging", Eur $J$ Nucl Med Mol Imaging, vol. 30, pp 1322 1329, 2003.

[24] A. Cot, C. Falcon, C. Crespo,C, J. Sempau,; D. Pareto, S. Bullich, F. Lomena, F. Calvino, J. Pavia and D. Ros, "Absolute quantification in dopaminergic neurotransmission SPECT using a Monte Carlo based scatter correction and fully 3 dimensional reconstruction", J Nucl Med, vol. 46, pp 1497 1504, 2005

[25] "Performance Measurements of scintillation cameras," NEMA Standards Publication NU 2001.

[26] E. Lage, J. J. Vaquero, A. Sisniega, S. Espana, G. Tapias, M. Abella, A. Rodriguez Ruano, J. E. Ortuno, A. Udias, and M. Desco, "Design and performance evaluation of a coplanar multimodality scanner for rodent imaging," Phys Med Biol, vol. 54, pp. $54275441,2009$. 